\title{
Classification of Finite Metric Spaces and Combinatorics of Convex Polytopes
}

\section{A. M. Vershik}

Received: 8 November 2014 / Accepted: 31 December 2014 / Published online: 20 January 2015 (C) Institute for Mathematical Sciences (IMS), Stony Brook University, NY 2015

\begin{abstract}
We describe the canonical correspondence between finite metric spaces and symmetric convex polytopes, and formulate the problem about classification of the metric spaces in terms of combinatorial structure of those polytopes.
\end{abstract}

Keywords Finite metric space $\cdot$ Convex polytope $\cdot$ Kantorovich-Rubinstein norm · Classification

\section{Introductory Remark}

We will discuss problems with very elementary formulations that concern the most popular notions in mathematics: metric spaces, convex geometry, combinatorics of polytopes and Kantorovich's optimal transportation. According to Arnold's classification, there are two ways to introduce a new subject: the first way (he called it the "Russian tradition") is to start with "the simplest non-trivial partial case"-I will use this approach. The second and the opposite tradition, which I also like very much (he called it "Bourbaki's tradition") is to start with an "extremely general case that is impossible to further generalize".

So my metric spaces will be finite, polytopes will be finite-dimensional etc. but all the notions and problems have infinite, infinite-dimensional, and continuous analogs.

To the memory of Dima Arnold.

This study supported by the Russian Science Foundation Grant 14-11-00581.

A. M. Vershik $(\bowtie)$

St. Petersburg Department of Steklov Institute of Mathematics, St. Petersburg State University,

Saint Petersburg, Russia

e-mail: avershik@gmail.com 


\section{General Problem}

Study and classify finite metric spaces according to combinatorial properties of their fundamental polytopes, associated with metric spaces in a canonical fashion. A more precise formulation follows.

In the rest of the paper, I explain the terminology that is used in the title and in the problem.

\section{Finite Metric Spaces, Canonical Polytopes, and Problems}

Let $(X, \rho),|X|=n$, be a finite metric space. We will write $V(X)$ for the vector space of all real-valued functions on $X$. The value of a function $v \in V(X)$ at a point $x \in X$ will be denoted by $v^{x}$. The space $V(X)$ can be naturally identified with the space of all formal linear combinations of elements of $X$. Under this identification, any element $x \in X$ identifies with the delta-function equal to 1 at $x$ and to 0 at all other points of $X$. Define the real vector space $V_{0}(X)$ as a subspace of $V(X)$ consisting of all $v \in V(X)$ with $\sum_{x \in X} v^{x}=0$.

Let us define the map $\delta: X \rightarrow V_{0}(X)$ taking an element $x$ to the function $\delta(x)=$ $\delta_{x} \in V_{0}(X)$ such that $\delta_{x}^{y}=-\frac{1}{n}$ for all $y \neq x$. Then we must have $\delta_{x}^{x}=\frac{n-1}{n}$.

The convex hull Conv $[\{\delta(x)\}, x \in X]$ of the image of this map is a $(n-1)$ dimensional simplex denoted by $\Sigma(X) \subset V_{0}(X)$ (this simplex is obtained from the standard coordinate simplex in $V(X)$ by the projection mapping $x \in X$ to $\delta(x)$ ).

The metric $\rho$ can be considered now as a metric on the vertices of the simplex $\Sigma(X)$, we use the same symbol $\rho$ to denote this metric. For every pair of distinct points $x$, $y \in X$, consider the vectors $e_{x, y} \in V_{0}$ defined by the formula:

$$
e_{x, y}=\frac{\delta_{x}-\delta_{y}}{\rho(x, y)} .
$$

This vectors will play a major role in what follows.

Definition 1 The fundamental polytope of a metric space $(X, \rho)$ is the convex polytope $R_{X, \rho}$ obtained as the convex hull of all vectors $e_{x, y}$, where $(x, y)$ run through all pairs of distinct points of $X$. The combinatorial type of $R_{X, \rho}$, i.e., the isomorphism class of the corresponding poset of faces, is called the combinatorial structure of the finite metric space $(X, \rho)$. Two finite metric spaces with the same combinatorial structures called similar metric spaces.

It is easy to see that the fundamental polytope $R_{X, \rho}$ is centrally symmetric (i.e., it coincides with its reflection in the origin). If $\rho(x, y)=1$ for any pair of distinct elements $x, y \in X$, then the fundamental polytope is the Minkowski sum of two simplices $\Sigma(X)$ and $-\Sigma(X)$.

In general, we consider the fixed set of rays $\left\{\lambda\left(\delta_{x}-\delta_{y}\right): \lambda>0\right\}$, which is independent of the metric, and choose one point in each ray with $\lambda=\frac{1}{\rho(x, y)}$ (this choice now depends on the metric). The fundamental polytope is the convex hull of all thus obtained points. 
A simple fact Mulleray et al. (2008) that characterizes the fundamental polytopes of finite metric spaces, is the following: given a symmetric function $\rho: X \times X \rightarrow \mathbb{R}_{>0}$, consider the polytope

$$
R_{X, \rho}=\operatorname{Conv}\left\{e_{x, y}=\frac{\delta_{x}-\delta_{y}}{\rho(x, y)}: \quad x \neq y, x, y \in X\right\}
$$

Then this function $\rho$ is a metric on $X$ if and only if no point $e_{x, y}$ belongs to the interior of convex hull of the other points.

The polytope $R_{X, \rho}$ is convex and central symmetric; therefore, it defines the Minkowski-Banach norm $\|\cdot\|_{\rho}$ in the real vector space $V_{0}(X)$, whose unit ball is by definition the polytope $R_{X, \rho}$. In the finite-dimensional case, this norm is the so called Kantorovich-Rubinstein norm. If $\rho(x, y)=1$ whenever $x \neq y$, then the corresponding fundamental polytope is the so called root polytope, and the corresponding Kantorovich-Rubinstein norm is the restriction to $V_{0}(X)$ of the $\ell^{1}$-norm in the space $V(X)$.

Thus we reduce the analysis of metric space to the convex geometry of fundamental polytope. Since the space $X$ is isometrically embedded into $V_{0}(X)$ (points of $X$ correspond to the vertices of the simplex $\Sigma(X)$ ), endowed with metric $\rho$ (see above) we can restore metric on $X$ using fundamental polytope.

Recall that the combinatorial type of a convex polytope is the isomorphism class of the partial ordered set form by the faces of the polytope, ordered by inclusion; the $f$-vector of a convex polytope is the finite tuple $\left(f_{0}, f_{1}, \ldots f_{n}\right)$, where $f_{0}=n$ is the number of vertices, $f_{1}$ is the number of edges, etc., $f_{n-1}$ is number of facets (i.e., faces of codimension 1) and, finally, $f_{n}=1$.

Our definition is functorial in the sense that each isometry of one metric space to another vector space produces an affine isomorphism of the corresponding fundamental polytopes.

We may say that the notion of the combinatorial type of metric spaces defines a natural stratification of the cone $\mathcal{M}_{n}$ of all distance matrices (i.e., real symmetric $n \times n$ matrices, whose entries are the values of a distance function defined on some finite metric space of cardinality $n$ ). Below we suggest to study this important stratification, more precisely, to solve the following problems.

Problem 1 1. Express the combinatorial structure of $(X, \rho)$ in terms of the metric $\rho$, i.e., find the $f$-vector of the corresponding fundamental polytope in terms of the metric $\rho$ itself - using linear inequalities on the values of metric etc.

2. Estimate the number of combinatorial structures for any given $n$ and study its asymptotic behavior as $n$ tends to infinity. The most interesting thing is to estimate the number of "open" (generic) types.

3. Provide sufficient conditions on two metric spaces to be similar.

It is well known that most finite metric spaces cannot be isometrically imbedded into a Euclidean or a Hilbert space (we say that these metrics do not have Euclidean type). The following question appears:

4. Describe the combinatorial types of metric spaces of Euclidean type. Do we obtain all combinatorial types? 
5. Does the stratification of the space of distance matrices into the combinatorial types is universal? Or, on the contrary, there are some restrictions on the topological types of the open components? ${ }^{1}$

\section{The Simplest Example: A Metric on a Cartan Subalgebra}

Consider a very degenerate metric space with $n$ points: $X=\mathbf{n}$, the set of all integers from 1 to $n$ with mutual distances between all pairs of distinct points equal to 1 : $\rho(i, j)=\delta_{i, j}$.

In this case, the simplex $\Sigma(X)$ is a regular Euclidean simplex. We can view it as $(n-1)$-simplex in a Cartan subalgebra of the Lie algebra $A_{n}$. From this viewpoint, the simplex is spanned by all positive simple roots $e_{i, i+1}, i=1, \ldots, n-1$, and one maximal root $e_{n, 1}$.

Proposition 1 Let $X=\boldsymbol{n}$ and $\rho(i, j)=\delta_{i, j}, i, j=1, \ldots, n$. Identify the vector space $V_{0}(X)$ with a Cartan subalgebra of the Lie algebra $A_{n}$. Then the fundamental polytope $R_{(X, \rho)}$ is the convex hull of all roots. The norm $\|.\|_{\rho}$ associated with the fundamental polytope coincides with the restriction of the $\ell^{1}$-norm on $V(X)$

$$
\left|v=\left(v_{1}, v_{2} \ldots v_{n}\right)\right|_{\ell^{1}}=\sum_{i=1}^{n}\left|v_{i}\right|,
$$

to the hyperplane $V_{0}(X) \subset V(X)$. Thus, the polytope $R_{X, \rho}$ in this case is the intersection of an $n$-dimensional octahedron with the hyperplane $V_{0}(X)$.

The corresponding norm $\|\cdot\|$ on the Lie algebra of skew-hermitian matrices with zero trace is the "nuclear norm", for which the norm of a matrix is the sum of the moduli of all its eigenvalues.

It is natural to call $R_{X, \rho}$ the "root polytope". An easy exercise is to find the $f$-vector in this case. For example, if $n=3$ and $\operatorname{dim}\left(V_{0}\right)=2$, then the fundamental polygon is a regular hexagon, and the norm is the hexagonal norm. For $n=4$, see the Fig. 1: the $f$-vector is equal to $(12,24,14)$, the facets are 8 regular triangles and 6 squares. For $n=3$, the group of symmetries of the fundamental polytope is the dihedral group $D_{6}=\mathbb{Z}_{2}<\mathbb{Z}_{6}$. For $n=4$, see the Fig. 2 .

Note that the group of symmetries of the root polytope includes the Weyl group (which is isomorphic to the symmetric group). Root polytopes were mentioned for other reason in Gelfand and Kapranov (1993).

\footnotetext{
1 A classification problem (in algebraic geometry, combinatorics, etc.) of a certain set of objects up to a certain equivalence relation is called a "universal problem" if all possible kinds of singularities or stratifications, say, of arbitrary semi-algebraic varieties can occur in the topology of equivalence classes.

A well-known example is Mnev's theorem on the universality of the combinatorial classification of convex polytopes in dimensions $\geq 4$. (see the papers by Vershik and Mnev (1988) and more recent literature).
} 


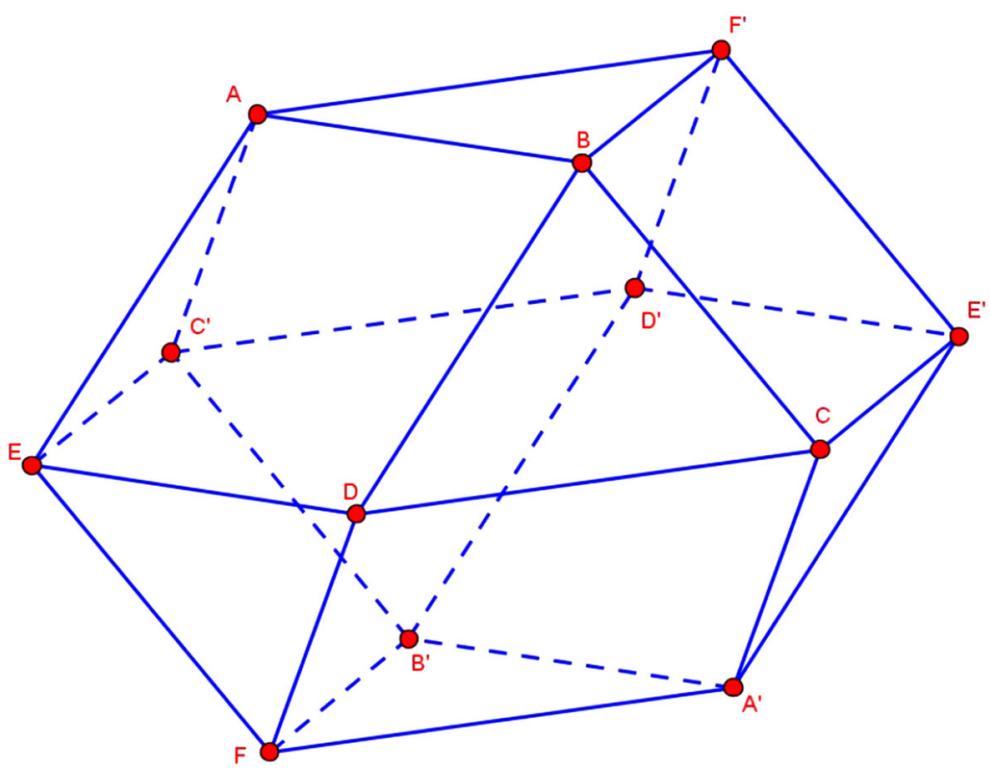

Fig. 1 The three-dimensional unit ball in Kantorovich-Rubinstein norm for 4 points space with metric $r(x$, $\mathrm{y})=1$ if $\mathrm{x} \neq \mathrm{y}$

Fig. 2 Octahedron-polytope which corresponds to metric space with points $(1,2,3,4)$ and metric: $r(i, j)=1, i, j=1,2,3$ $\mathrm{i} \neq \mathrm{j}, \mathrm{r}(\mathrm{i}, 4)=1 / 2, \mathrm{i}=1,2,3$

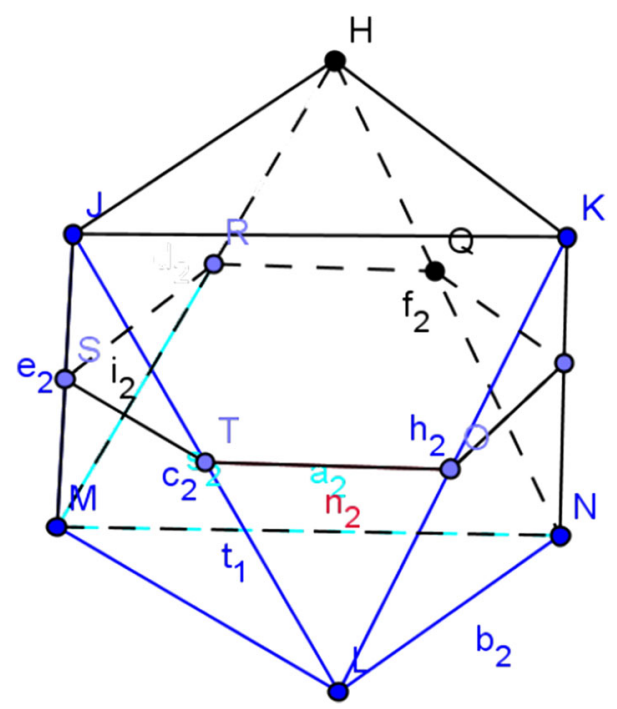

\section{Why Such Combinatorial Types? The Kantorovich Metric and Geometry of the Transportation Problem}

Why is the definition of the fundamental polytope $R_{X, \rho}$ associated with a finite metric space $(X, \rho)$ natural? The justification is as follows. We want to define a natural metric $\bar{\rho}$ on $V_{0}(X)$ that extends the metric $\rho$ on $X$. The latter is identified with the 
set of vertices of the simplex $\Sigma(X)$ so that the distance between two points $x$ and $y$ in $X$ coincides with the distance between the corresponding vertices $v_{x}$ and $v_{y}$ of the simplex $\Sigma(X)$. In another words, we want to define a norm $\|\cdot\|$ in $V_{0}(X)$ with the property $\left\|e_{x, y}\right\|=\rho(x, y)$. There are many such extensions but there is a maximal one:

Theorem 1 (Mulleray et al. 2008) The norm $\|\cdot\|_{\rho}$, called the Kantorovich-Rubinstein norm, is the unique maximal extension of $\rho$ : all other norms possessing this extension property are less that this one because the fundamental polytope is contained in the unit balls of all such norms.

The direct description of an extension of the metric $\rho$ to the whole simplex $\Sigma(X)$ is a classical definition by Kantorovich of his transportation metric. This definition is well known in the mathematical economics and in linear programming but there were no publications [before Mulleray et al. (2008), see comments below], in which characteristic properties of fundamental polytopes are discussed and serious combinatorial investigations are conducted. Recall, for the sake of completeness, the classical definition of the Kantorovich transportation metric, which is equivalent to our definition above.

Consider the positive part $v(+)$ and the negative part $v(-)$ of the vector $v$. The vector $v(+)$ is defined as the componentwise maximum of $v$ and the zero vector, and the vector $v(-)$ is defined as the componentwise minimum. Evidently, we have $v=v(+)+v(-)$. Thus we have two nonnegative vectors $v(+)=u$ and $v(-)=w$ with equal sums of coordinates.

Then classical definition is

$$
\bar{\rho}(u, w)\left(=\|u-w\|_{\rho}\right)=\min _{\psi \in \Psi} \sum \rho(x, y) \psi_{x, y},
$$

where $\Psi=\left\{\psi_{x, y}\right\}$ is the convex set of all matrices $\psi$ with the following properties:

$$
\sum_{x} \psi_{x, y}=u^{x} ; \quad \sum_{y} \psi_{x, y}=w^{y}, \quad \psi_{x, y} \geq 0, \quad x, y \in X .
$$

Here $u^{x}$ is the coordinate of the vector $u$ corresponding to the point $x \in X$, and similarly for $w$.

For the history of the Kantorovich metric, see Vershik (2013) and references therein. For some further development and applications of the finite-dimensional geometry of this metric, see Vershik (2014).

The last question is also of Arnold's style (see e.g. Arnold 2006): in our definition of the Kantorovich metric, we used only the root system $A_{n}$. My question is: what are the analogs of this definition (and maybe even of the transportation problem!) for other series of Lie algebras and other Weyl groups.

\section{References}

Arnold, V.: Statistics of the symmetric group representations as a natural science question on asymptotic of young diagrams. In: Representation Theory, Dynamical Systems, and Asymptotic Combinatrics. Advances in the Mathematical Sciences Series 2, vol. 217, pp. 1-7. American Mathematical Society, Providence (2006) 
Gelfand, I., Kapranov, M.: On the dimension and degree of the projective dual variety: a $q$-analog of the Katz-Kleiman formula. In: Corwin, L., Gelfand, I., Lepowsky, J. (eds.) The Gelfand Mathematical Seminars 1990-1992, pp. 27-33. Birkhauser, Boston (1993)

Mulleray, J., Petrov, F., Vershik, A.: Linearly rigid metric spaces and the embedding problem. Fundam. Math. 199(2), 177-194 (2008)

Vershik, A.: Long history of the Monge-Kantorovich transportation problem. Math. Intell. 35(4), 1-9 (2013)

Vershik, A.: The problem of describing central measures on the path spaces of graded graphs. Funct. Anal. Appl. 48(4), 26-46 (2014) (In Russian)

Vershik, A., Mnev, N.: Topology and geometry—Rokhlin seminar. Lect. Notes Math. 1346, 557-581 (1988) 